\title{
Combining Simulations and Machine Learning for Efficient Prediction of Process Parameters Evolution in Injection Moulding
}

\author{
Albert $\mathrm{ABIO}^{\mathrm{a}, 1}$, Francesc BONADA ${ }^{\mathrm{a}}$ and Oriol PUJOL ${ }^{\mathrm{b}}$ \\ ${ }^{a}$ Eurecat, Centre Tecnològic de Catalunya, Unit of Applied Artificial Intelligence, \\ Cerdanyola del Vallès, Spain \\ ${ }^{\mathrm{b}}$ Department de Matemàtiques i Informàtica, Universitat de Barcelona, Barcelona, \\ Spain
}

\begin{abstract}
In recent years, the emerging technologies in the context of Industry 4.0 have led to novel approaches in process monitoring and control, such as the introduction of Reinforcement Learning and Digital Twins. Consequently, large amounts of data, precise modelling and exhaustive simulations are required. The aim of this work is to propose a methodology based on the technique of backward selection to reduce the number of reference points in the simulation stage of manufacturing processes, enhancing the efficiency of data generation and the simplicity of the simulations. The methodology is proved in the particular case of plastic injection moulding simulations.
\end{abstract}

Keywords. Industry 4.0, Backward Selection, Machine Learning, Manufacturing Simulation, Plastic Injection Moulding, Node Reduction

\section{Introduction}

Simulations are widely used in several fields, but one of their most relevant applications is in manufacturing, where they are established as a powerful tool for evaluating, validating and optimizing design and manufacturing processes [1]. Additionally, they also allow the digitalization of the manufacturing system, being able to provide knowledge without perturbing the real system.

The current manufacturing paradigm is drifting towards data-driven systems. These define the core of what is known as Industry 4.0. Under this paradigm, new tools and techniques based on data and artificial intelligence are introduced as drivers for innovation that impact in the productivity. The treatment with advanced analytic techniques of the information acquired during the manufacturing process allows gathering complex and precise knowledge about the system as well as to enable the deployment of predictive quality and maintenance protocols [2]. Machine Learning (ML) techniques have in-

\footnotetext{
${ }^{1}$ E-mail: albert.abio@eurecat.org
} 
creased their relevance to address manufacturing problems, since they can deal with high dimensional data and they are able to uncover and model nonlinear relationships [3].

Supervised and unsupervised ML methods have been the most usual techniques applied in manufacturing to date, although Reinforcement Learning (RL) has started to show its high potential in this field [4].

The Digital Twin (DT) is one of the latest technology trends in the framework of Industry 4.0. It consists in a digital version of a physical entity that exists in a virtual space and that it is in constant interaction with the physical space [5]. At present, there is a strong interest in the research and development of DTs, since they are able to use the information of a real-world system into a virtual system, enhancing the knowledge of the operating status and optimizing its performance.

The implementation of DTs requires difficult modelling through exhaustive simulations. Moreover, to effectively apply ML techniques, and in particular RL strategies, a large amount of data needs to be generated. In the particular case of manufacturing, the experimental data is costly and involves the waste of raw materials, making the acquisition of data under non-productive conditions difficult or unfeasible. In this scenario simulations become the main source of data. However, generating data using simulations models of the manufacturing processes is time consuming and computationally expensive. The direct consequence is the difficulty in the development of DTs or the application of RL in manufacturing. A possible solution to this problem is to combine simulations with ML predictive techniques to enhance and speed up the virtual data generation.

The approach of combining simulations and ML can be used to build simpler physical models, which reduce the computational resources that complete models require. These kind of models are usually called surrogate models or response systems, which represent the system in a simpler but representative way. Hence, the data is obtained efficiently without a relevant lost of knowledge about the system [6].

In this work, we explore the hybridization of physical phenomenological simulations with ML prediction techniques and we develop a methodology to increase the simulation efficiency, in the particular case of plastic injection moulding. The goal of this hybrid model is to combine ML predictions with a reduced number of simulation nodes with the goal of describing a more complete phenomenon. In the proposed study the best points to simulate in order to obtain a reliable description of the process are identified. Additionally, an adapted backward selection methodology is used for node selection task.

\section{Plastic Injection Moulding Simulation}

Plastic injection moulding consists in the injection of melted plastic into a mould where it cools down and solidifies to acquire the desired shape [7]. The process comprises different steps: First, the plastic is melted inside a barrel applying temperature, pressure and shear using a rotational screw. Then, the injection of the required plastic into the mould is carried out through a shot. Afterwards, a packing pressure is applied to guarantee part dimensional characteristics.

In this study, the objective is to increase the efficiency of data generation in plastic injection moulding process. For this reason, simulations of the process have been performed with the Moldex3D mold flow analysis commercial software [8], based on Finite Element Analysis (FEA). The studied part is a cap injected in a mould cavity. In Figure 


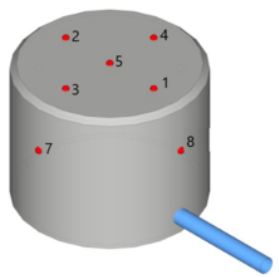

(a)

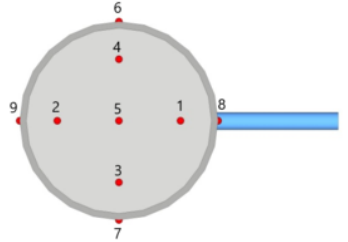

(b)

Figure 1. Sketch of the cap form different perspectives. The melted plastic enters through the blue runner. The red dots are the sensed points and the numbers are used to identify the different pressure sensors.

Table 1. Values of the packing pressure $(P P)$ and the injection speed $(v)$ for the 15 generated configurations. The marked configurations (*) are used for testing.

\begin{tabular}{|c|c|c|c|c|c|}
\hline Configuration & $P P(M P a)$ & $v(\mathrm{~mm} / \mathrm{s})$ & Configuration & $P P(M P a)$ & $v(\mathrm{~mm} / \mathrm{s})$ \\
\hline conf 1 & $P P_{r e f}+0.1 P P_{r e f}$ & $v_{r e f}$ & conf 9 & $P P_{\text {ref }}$ & $v_{r e f}-6$ \\
\hline $\operatorname{conf} 2 *$ & $P P_{r e f}+0.2 P P_{r e f}$ & $v_{r e f}$ & conf 10 & $P P_{r e f}$ & $v_{r e f}-4$ \\
\hline conf 3 & $P P_{r e f}+0.3 P P_{r e f}$ & $v_{r e f}$ & conf $11 *$ & $P P_{r e f}$ & $v_{r e f}-2$ \\
\hline conf 4 & $P P_{r e f}-0.1 P P_{r e f}$ & $v_{\text {ref }}$ & conf 12 & $P P_{\text {ref }}$ & $v_{r e f}+2$ \\
\hline $\operatorname{conf} 5^{*}$ & $P P_{r e f}-0.2 P P_{r e f}$ & $v_{r e f}$ & conf 13 & $P P_{r e f}$ & $v_{r e f}+4$ \\
\hline conf 6 & $P P_{r e f}-0.3 P P_{r e f}$ & $v_{r e f}$ & conf $14 *$ & $P P_{\text {ref }}$ & $v_{\text {ref }}+6$ \\
\hline $\operatorname{conf} 7$ & $P P_{r e f}$ & $v_{r e f}$ & conf 15 & $P P_{r e f}$ & $v_{r e f}+8$ \\
\hline $\operatorname{conf} 8$ & $P P_{r e f}$ & $v_{r e f}-8$ & & & \\
\hline
\end{tabular}

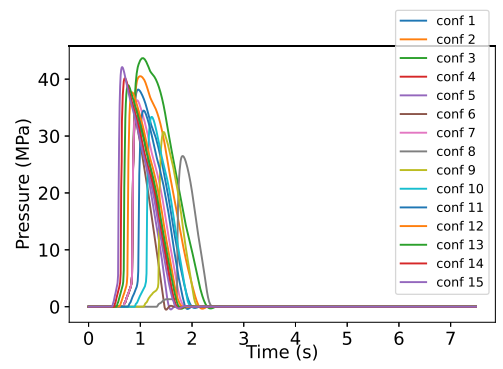

(a)

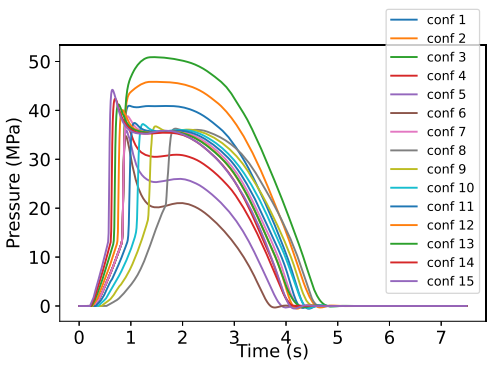

(b)

Figure 2. Pressure evolution in the simulation of the process for all the configurations in (a) SN5 and (b) SN8.

1 , the geometry of the cap is illustrated. Moreover, nine virtual or simulated sensors have been displayed in the geometry: SN1 - SN9. The sensors measure the cavity pressure evolution in these nine selected points, since it has been identified in the literature as one of the most relevant variables for the quality of the final product [7].

To extend the study to different conditions, several configurations have been generated changing two parameters of the simulations: the injection speed $(v)$ of the plastic into the mould and the value of the packing pressure $(P P)$, as shown in Table 1. These parameters have been modified around their nominal working values $v_{r e f}$ and $P P_{r e f}$. The result are 15 configurations representing the conditions of a real-environment. The simulations last in the range of 40 to $50 \mathrm{~min}$ per configuration.

The output of the simulations is the evolution of the cavity pressure exerted by the melted plastic in the nine points where the sensors are located. Initially, the sampling 
time in Moldex3D for the pressure data is different for each simulated configuration. In order to homogenize them, we apply a resampling each $0.01 \mathrm{~s}$ to homogenize all the configurations, obtaining 750 samples per configuration. In Figure 2, the pressure evolution behavior is shown for all the different configurations in two of the sensed points. It is worth noting the heterogeneity of the results for different sensors and configurations.

\section{Experimental Setup and Methodology}

In this section, we present the experimental set-up that aims to understand up to which extend simulation nodes can be replaced with ML predictions. To this end, two experiments are carried out: An individual assessment of each sensor and a global assessment for the complete set of sensors. In this article, we adopt a methodology that systematically replaces simulated sensors by predicted ones by means of a backward search. In particular, the sensors selected for testing purposes are such that they define the worst case scenario, ensuring that any other choice would achieve better scores.

\subsection{Experimental Setup}

From the nine different simulated sensors, three of them will be used for assessing the quality of the prediction system. The three selected target pressure sensors will be predicted using the remaining simulated sensors data. Additionally, to ensure the generalization of the algorithm on independent test data, the set of 15 configurations is split in 11 configurations for training and a 4 configurations for testing (see values in Table 1 marked with a star). The test configurations have been chosen to be intermediate values of the simulation parameters (injection speed and packing pressure).

To select the target testing sensors, we use the concept of similarity between the samples of the time series of the pressure sensors. Then, we compute the mean similarity [11] between the pressure curves of the sensors $\operatorname{tsim}(X, Y)$ as follows,

$$
\operatorname{tsim}(X, Y)=\frac{1}{n} \sum_{i=1}^{n} \operatorname{numSim}\left(x_{i}, y_{i}\right)
$$

where $X=x_{1}, \ldots, x_{n}$ and $Y=y_{1}, \ldots, y_{2}$ are time series of two pressure sensors and $\operatorname{numSim}\left(x_{i}, y_{i}\right)=1-\frac{\left|x_{i}-y_{i}\right|}{\left|x_{i}\right|+\left|y_{i}\right|}$ is the similarity between two samples in the same instant of time. The operation range of $\operatorname{tsim}(X, Y)$ lies in the interval $[0,1] \cdot \operatorname{tsim}(X, Y)=1$ refers to two identical pressure curves.

Figure 3 is the result of computing the mean similarity between the different sensors averaging for the 4 test configurations. This result drives the selection of the less similar sensors for the prediction, with the intention to use non-trivial cases to validate the presented methodology.

Comparing Figure 3 with the localization of the sensors in the cap (Figure 1), we can observe the symmetry relations displayed in the Mean Similarity Matrix between the sensors SN3 and SN4 and the sensors SN6 and SN7. These sensors will be discarded for prediction because they will not suppose a difficulty for the algorithm, that will use the corresponding symmetric sensor to obtain a very good prediction. The remaining 


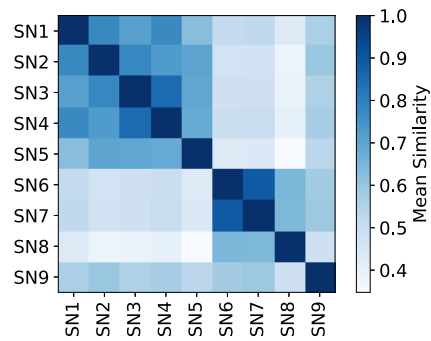

Figure 3. Mean Similarity Matrix averaged over the 4 test configurations. The spatial regions of the cap can be differentiated in this matrix. The first 5 sensors are in the superior part of the cap and the last 4 in the lateral.

sensors are not symmetric due to the position of the runner. The three sensors with less similarity are SN5, SN8 and SN9. Due to the central position of SN5 in the cap, we are interested in the real value of the cavity pressure in that point. We prefer not to include SN5 in the set of target sensors and replace it with SN2, which has no symmetries in the cap geometry. Summarizing, in a first approach, the sensors SN2, SN8 and SN9 will be predicted using the values of the rest of pressure sensors. As mentioned, this defines a worst case scenario.

\subsection{Backward Selection Methodology}

In the previous section we have defined a set of three target sensors that will be predicted using the data from the six remaining sensors. This means that in future simulations six points will still have to be sensed. In order to minimize the number of sensed points for future simulations and explore to which extend these can be replaced by ML predictions we propose to use a methodology based on the technique of backward selection [12].

The technique consists in the elimination of the input features of a ML algorithm, using a metric that allows to decide which feature is the best to drop in a greedy manner. Starting from a set of $k=1, \ldots, M$ input features and $i=1, \ldots, L$ test configurations, the elimination of features is carried out through the following iterative process:

1. Use the current number of features $M$ to predict the target.

2. Compute the error metric for each of the test configurations, the Mean Squared Error (MSE) in our case.

3. For all the $k=1, \ldots, M$ current features:

(a) Predict the target without using the feature $k$.

(b) Compute the error metric for all the test configurations.

(c) Calculate $\operatorname{diff}_{i}^{M-1, M}(k)$, the error difference with and without feature $k$ for each test configuration as follows,

$$
\operatorname{diff}_{i}^{M-1, M}(k)=\operatorname{MSE}_{i}^{M-1}(k)-\operatorname{MSE}_{i}^{M}
$$

(d) Perform the weighted average $t_{\text {conf }}^{M}(k)$ described in the following Eq. (3) of the differences over the test configurations for the eliminated feature $k$.

$$
t_{\text {conf }}^{M}(k)=\sum_{i=1}^{L} c_{i}(k) \operatorname{diff}_{i}^{M-1, M}(k)
$$




$$
\text { where } c_{i}(k)=\frac{\operatorname{MSE}_{i}^{M}}{\sum_{j=1}^{L} \operatorname{MSE}_{j}^{M}}
$$

4. Select the smaller value of $t_{\text {conf }}^{M}(k)$ and drop the corresponding feature $k$.

5. Repeat the process with the new set of features of size $M=M-1$.

The process ends when the number of desired features is reached or when the error overcomes a given threshold. Observe that the value of $\operatorname{diff}_{i}^{M-1, M}(k)$ may be negative if the elimination of the feature $k$ improves the prediction algorithm performance. The proposed methodology takes into account the value of error metric for each test configuration to decide which is the best feature to drop, since it is preferable to optimize the predictions of the configurations that have a higher error.

\section{Results and Discussion}

\subsection{Baseline Prediction Results}

The reduction of the sensed points is realized with the application of a ML regression algorithm that uses as input data coming from a few locations to predict the rest of the points. Before that, we perform an algorithm comparison in order to know the prediction capability of some regression algorithms to all the available data. Therefore, we will randomly merge the data from all the configurations, obtaining a dataset composed by $750 \times 15$ samples and 9 features. Selecting a target sensor to predict and using all the others for training, we will implement a 10-Fold CV [9] to choose a candidate algorithm.

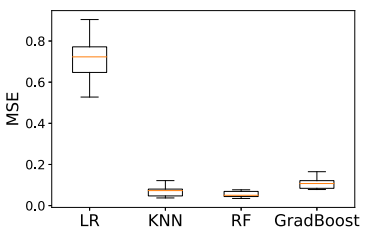

(a)

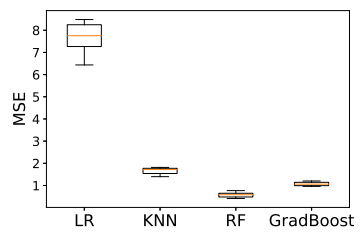

(b)

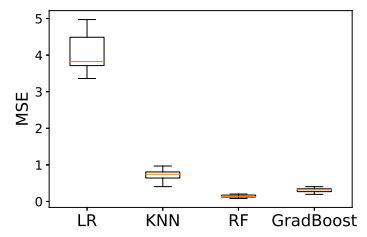

(c)

Figure 4. Average Mean Squared Error (MSE) of a 10-Fold CV for algorithm comparison between Linear Regression (LR), k-Nearest Neighbours Regressor (KNN), Random Forest Tree Regressor (RF) and Gradient Boosting Regressor (GradBoost). Target predicted sensor: (a) SN2. (b) SN8. (c) SN9.

Figure 4, shows the error performance comparison of four different regression techniques applied to the complete dataset. Random Forest Tree Regressor [10] achieves a lower error rate and will be used for the rest of the experiments.

\subsection{Individual Sensor Reduction Assessment}

The purpose of this study is to demonstrate the feasibility of achieving an important reduction of the number of sensed points without having a high impact in the prediction error. As explained in the experimental setup section, we will reduce the number input sensors used to predict the set of three target sensors, by applying the methodology presented in section 3.2. 


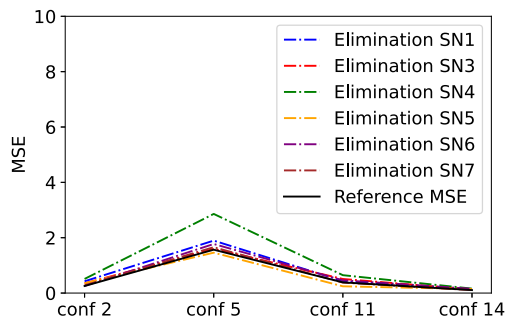

(a)

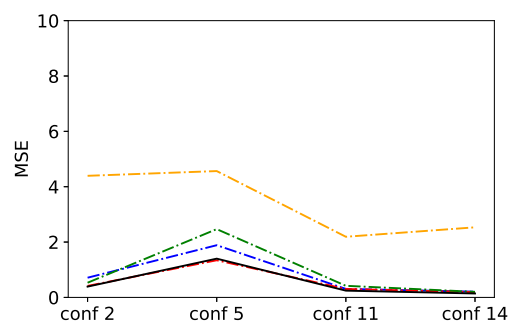

(c)

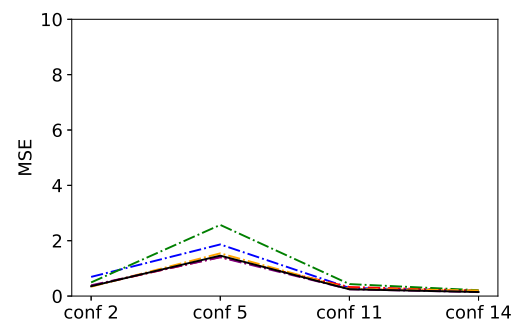

(b)

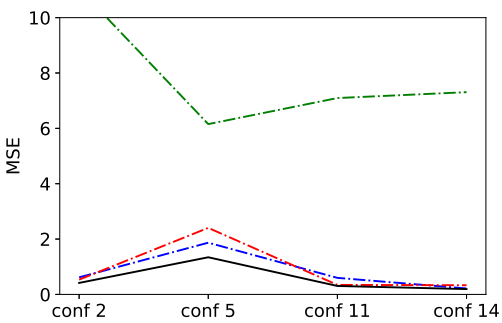

(d)

Figure 5. MSE in test configurations for target SN9. The black curve indicates the reference MSE from the previous step of the elimination process and the colored curves indicate the MSE with the drop of one of the sensors. (a) 5 sensor selection. (b) 4 sensor selection. (c) 3 sensor selection. (d) 2 sensor selection.

The different steps of the backward selection process are displayed in Figure 5, where the MSE for each test configuration is represented when we eliminate the input sensors. The curve of reference MSE does not suffer a relevant variation during the stages of the process, meaning that the prediction capability of the algorithm remains despite the discarded sensors. It refers to the MSE computed with $M$ sensed points and it is used to evaluate the predictions with $M-1$ sensed points through the use of Eq. (3).

Figure 6 shows the result of the selection process for each one of the target sensors. The evolution of the mean MSE of the 4 test configurations allows to identify a threshold in three input sensors. Below this threshold, the use of less input sensors induces the error metric to start having a relevant increase. By inspecting these values, Table 2 shows the three best input sensors for individually predicting each target sensor.

Table 2. Best input sensors for the corresponding target sensors.

\begin{tabular}{|cccc|}
\hline Target sensor & SN2 & SN8 & SN9 \\
\hline Best input sensors & SN1, SN3, SN6 & SN6, SN1, SN5 & SN6, SN4, SN1 \\
\hline
\end{tabular}

\subsection{Global Sensor Reduction Assessment}

The results in the previous section 4.2 open the possibility of reducing the number of sensed points, showing that each individual sensor can be predicted using 3 sensed points 


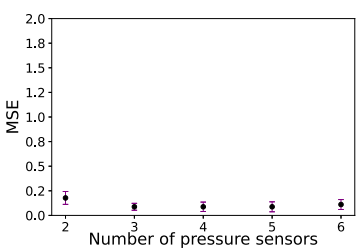

(a)

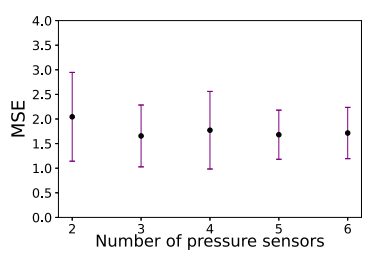

(b)

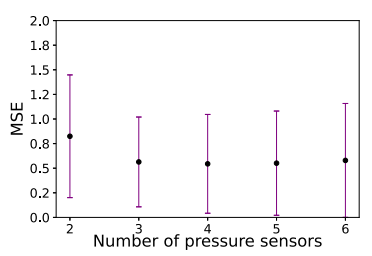

(c)

Figure 6. Evolution of the mean and the standard deviation over configurations with the number of input sensors used in the prediction. (a) SN2. (b) SN8. (c) SN9.

without a relevant effect in the error. However, the results show that different nodes require of different sensors for a good performance. In this subsection we consider whether a small common set of sensors may suffice for predicting all targets.

In order to do so, we will select the most repeated input sensors to predict all the target sensors. With the information of Table 2, we can identify that SN1 and SN6 are important for the prediction of the 3 target sensors. Additionally, we will choose the SN5, since it has a central position in the cap geometry (Figure 1). Accordingly, the final set of input sensors is formed by SN1, SN5 and SN6.

The defined final set of input sensors is used to predict the pressure of the target sensors. In Figure 7, the error metric MSE is compared when the prediction is done with 6 or 3 input sensors. If we use the individual set of 3 sensors of Table 2, we achieve a decrease of the error in most of the cases. Elseways, the use of the common set of sensors leads to a higher prediction error, but it allows to reduce the number of sensed points in the simulations. Moreover, the common set of sensors is not only able to predict the target sensors but also it yield good predictions for all the remaining sensors that the methodology has discarded. Figure 7d shows these results and it demonstrates the generalization capability of the proposed methodology. In the framework of industrial problems, it can be useful to include previous knowledge in a human - AI interaction that aims to help the global system performance, as shown including SN5 in the common set of input sensors.

As a final result, Figure 8 shows simulated and predicted pressure curves of the target sensors for a certain configuration. As observed in Figure 7, the variability of the accuracy of the prediction is highly dependent on the target sensor and the test configuration. Figure $8 \mathrm{c}$ shows the worst case prediction. Regardless of not being a perfect prediction, some relevant features of the injection moulding process such as the maximum value of the curve or the duration of the different stages are correctly characterized [7]. This result is of high importance as in manufacturing processes, the control a few relevant process variables is enough to determine the global performance of the system.

\section{Conclusions}

In this work, we have studied the hybridization of simulations with ML predictions applied to increase efficiency in a plastic injection moulding simulation process. The main results show that sensor nodes can be replaced with predicted versions using a very small set of real simulated data. This has been tested in experiments where process parameters 


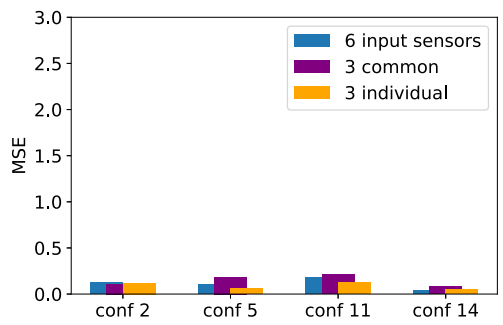

(a)

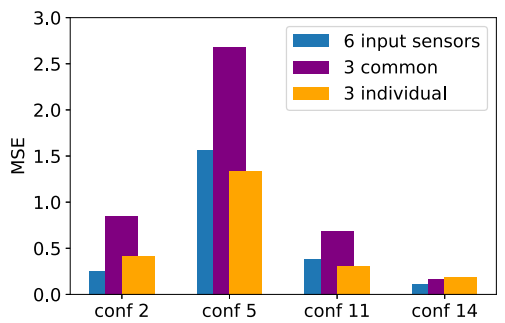

(c)

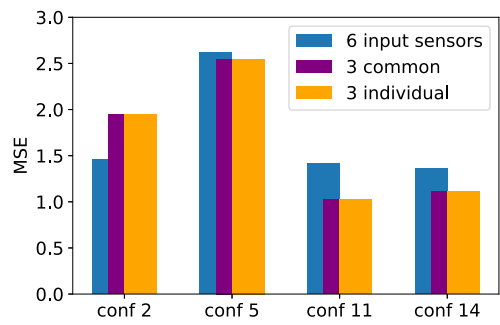

(b)

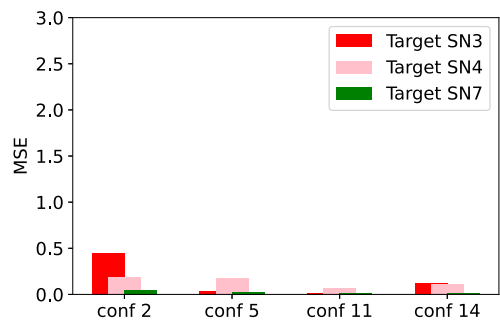

(d)

Figure 7. MSE comparison using 6 input sensors, the final set of 3 input sensors or the best 3 selected sensors for each individual target sensor. Target sensor: (a) SN2. (b) SN8. (c) SN9. (d) MSE of the prediction of the sensors SN3, SN4 and SN7 as target with the selected set of 3 input sensors.

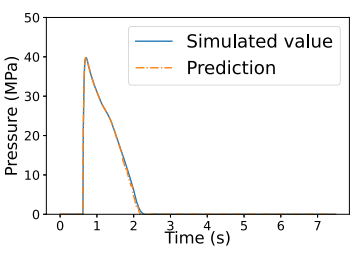

(a)

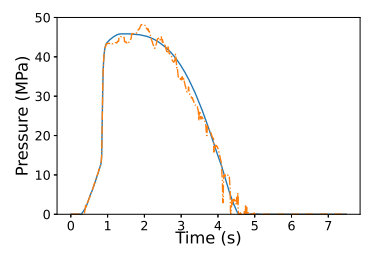

(b)

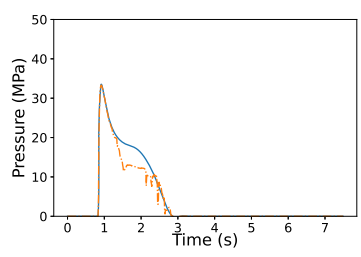

(c)

Figure 8. Comparison example between the simulated and the predicted temporal evolution of the pressure using the 3 selected input sensors. (a) Target SN2, conf 14. (b) Target SN8, conf 2. (c) Target SN9, conf 5.

(packing pressure and injection velocity) are different from those used for the data in the training set. Although in specific configurations the results may be worsened due to the reduction in the sensed nodes, the predictions obtained still preserve the critical process variable values, namely, maximum pressure value, duration of the process stages, etc. This is an important result because the global performance of the system is highly dependent on these values.

Although the proposed methodology has been used for a particular manufacturing problem, it can be extended to other applications. For instance, in the generation of the experimental data, it may be interesting to use less sensors to obtain the same amount of data due to the economical impact of these devices. On the other hand, in the simulation 
field, the adaptation of the methodology for a more general scenario with a larger number of points to reduce may still be a challenge to address. The generation of more complex similar surrogate models could have a high impact in the efficiency of simulations. The decrease of the nodes used for simulation could lead to lower simulation times, which are an issue in some common simulation methods like Finite Element Method (FEM).

Finally, future research focus on increasing the efficiency of the data generation by means of more complex surrogate models that are based on the hybridization of simulations and ML. In the context of Industry 4.0, this will immediately boost the development of promising data-driven technologies that need a large quantity of data to be implemented, such as the DTs or RL.

\section{Acknowledgements}

Albert Abio is a fellow of Eurecat's "Vicente López" PhD grant program. This work has been partially funded by the Spanish project PID2019-105093GB-I00 (MINECO/ FEDER, UE).

\section{References}

[1] Mourtzis D, Doukas M, Bernidaki D. Simulation in Manufacturing: Review and Challenges. Procedia CIRP. 2014; 25: 213-229.

[2] Tao F, Qi Q, Liu A, Kusiak A. Data-driven smart manufacturing. Journal of Manufacturing Systems. 2018; 48: 157-169.

[3] Wuest T, Weimer D, Irgens C, Thoben KD. Machine learning in manufacturing: advantages, challenges, and applications. Production and Manufacturing Research. 2016; 4(1): 23-45.

[4] Oliff H, Liu Y, Kumar M, Williams M, Ryan M. Reinforcement learning for facilitating human-robotinteraction inmanufacturing. Journal of Manufacturing Systems. 2020; 56: 326-340.

[5] Tao F, Zhang H, Liu A, Nee, AY. Digital twin in industry: State-of-the-art. IEEE Transactions on Industrial Informatics. 2018; 15(4): 2405-2415.

[6] von Rueden L, Mayer S, Sifa R, Bauckhage C, Garcke J. Combining Machine Learning and Simulation to a Hybrid Modelling Approach: Current and Future Directions. International Symposium on Intelligent Data Analysis. 2020: 548-560.

[7] Kurt M, Kamber OS, Kaynak Y, Atakok G, Girit O. Experimental investigation of plastic injection molding: Assessment of the effects of cavity pressure and mold temperature on the quality of the final products. Materials and Design. 2009; 30(8): 3217-3224.

[8] https://www.moldex3d.com/

[9] Arlot S, Celisse A. A survey of cross-validation procedures for model selection. Statistics surveys. 2010; 4: 40-79.

[10] Ho TK. Random decision forests. In: Proceedings of 3rd International Conference on Document Analysis and Recognition; 1995 Aug 14-16; Montreal, QC. IEEE; 1995. p.278-282.

[11] Cassisi C and Montalto, $P$ and Aliotta $M$ and Cannata, A and Pulvirenti A. Similarity measures and dimensionality reduction techniques for time series data mining. In: Karahoca A, editor. Advances in Data Mining Knowledge Discovery and Applications. Rijeka (HR): IntechOpen; 2012. p. 71-96.

[12] Sorjamaa A, Hao J, Reyhani N, Ji Y, Lendasse A. Methodology for long-term prediction of time series. Neurocomputing. 2007; 70: 2861-2869. 